\title{
Métodos de siembra de Pennisetum sp. "maralfalfa" en el rendimiento forrajero
}

Sowing methods of Pennisetum sp. "maralfalfa" in forage yield

Roberto Carlos Quispe Varillas, Rufino Maximo Maguiña-Maza

\section{RESUMEN}

Objetivo: Evaluar la influencia del método de siembra de Pennisetum sp. "maralfalfa" en el rendimiento forrajero. Métodos: Se utilizaron un área de cultivo de $4000 \mathrm{~m}^{2}$, con 5600 semillas sembradas con diferentes grados de inclinación: $0^{\circ}, 25^{\circ}$ y $45^{\circ}$. Las variables evaluadas fueron rendimiento forrajero, peso de planta, número de tallos y altura de planta. Los datos fueron analizados utilizando el análisis de varianza de un diseño completamente al azar. Resultados: La siembra a $25^{\circ}$ de inclinación tuvo mejores resultados en el rendimiento forrajero, peso de planta, número de tallos y altura de planta. Conclusión: La siembra a $25^{\circ}$ de inclinación mostró mejores valores de cosecha.

Palabras clave: Maralfalfa, método de siembra, rendimiento de forraje, grado de inclinación.

\section{ABSTRACT}

Objective: Evaluate the influence of the planting method of Pennisetum sp. "Maralfalfa" in forage yield. Methods: A cultivation area of $4000 \mathrm{~m} 2$ was used, with 5600 seeds sown with different degrees of inclination: $0^{\circ}, 25^{\circ}$ and $45^{\circ}$. The variables evaluated were forage yield, plant weight, number of stems and plant height. The data were analyzed using the analysis of variance of a completely randomized design. Results: Planting at $25^{\circ}$ inclination obtained better results in forage yield, plant weight, number of stems and plant height Conclusions: Planting at $25^{\circ}$ inclination selected better harvest values.

Keywords: Maralfalfa, sowing method, forage performance, degree of inclination. 


\section{INTRODUCCIÓN}

La implantación de los cultivos forrajeros megatérmicas es un factor de vital importancia para la vida productiva de la pastura, principalmente del género Pennisetum, con un periodo de crecimiento de las yemas (brotes), emergencia y establecimiento de la planta luego de la siembra, fase que pueda prolongarse entre tres a cinco meses (Calzada-Marín, Enríquez-Quiroz, Hernández-Garay, Ortega-Jiménez, y MendozaPedroza, 2014 y Oquendo, 2002) y dependerá de las condiciones de crecimiento que disponga la planta y el clima (Ayala, 1990).

Para la siembra se requiere semillas provenientes de tallos maduros, gruesos, en pedazos o cañas enteras (INIAP, 1999). La propagación por semillas vegetativas a través de estacas con tres nudos viables, sembradas en los surcos en forma inclinada con dos nudos dentro del suelo y una fuera de ella, desarrollan en la parte aérea nuevas plantas y raíces en el suelo (Borbor, 2013). También, hay siembras de semillas vegetativas bajo suelo (chorro continuo) que responde comparativamente con otros métodos de siembra (Molina, 2005).

Los mejores suelos que se ubican en los valles costeros son utilizados para la producción agrícola que son mucho más rentables que la producción de pastos y forrajes. Sin embargo, en la costa hay grandes extensiones de tierras marginales y eriazas que podrían servir para ampliar el piso forrajero para la producción pecuaria local.

El cultivo de Pennisetum sp. "maralfalfa" es poco conocido por parte de los ganaderos, por lo cual es muy importante difundir la información de un nuevo material genético que ofrece buen rendimiento de proteína y de biomasa forrajera a diferencia con otras especies del género pennisetum. La siembra de esta especie en la zona es igual a la caña de azúcar (chorro continuo o a $0^{\circ}$ grados de inclinación) o inclinadas. Debido a esta razón, en el presente estudio se realizó la siembra en suelo eriazo con el objetivo de evaluar la influencia de los métodos de siembra con semilla vegetativa en el rendimiento forrajero de la "maralfalfa".

\section{MATERIALY MÉTODOS}

El estudio se realizó en el establo Piamonte SAC, ubicado en el sector Guayabal - Pampa de Jopto, distrito Santa María, provincia de Huaura. Utilizando un área de $4000 \mathrm{~m}^{2}$, a $85 \mathrm{msnm}$ de altitud. La temperatura media en el distrito oscila entre $23^{\circ} \mathrm{C}$ en verano y $15^{\circ} \mathrm{C}$ en invierno. Según el análisis el suelo, es de característica arenoso franco con $\mathrm{pH}$ de 8,5, conductividad eléctrica de $0,46 \mathrm{mS} / \mathrm{cm}$ y contenido de materia orgánica de $0,7 \%$.
Las labores culturales realizadas en la siembra fueron limpieza, nivelación y preparación del suelo, utilizando un arado vertedera a una profundidad de 30 a $40 \mathrm{~cm}$. Se utilizó semillas vegetativas (5 600 estacas aprox.) de tres nudos cada uno, con promedio de $120 \mathrm{~g}$ de peso y $50 \mathrm{~cm}$ de longitud, provenientes de plantas con 5 meses de crecimiento. La siembra se realizó entre $0,90 \mathrm{~m}$ de distancia entre surcos y 0,83 $\mathrm{m}$ de distancia entre semillas, plantando en diferentes inclinaciones $(0,25$ y 45 grados $)$. La siembra del primer tratamiento fue con tres nudos bajo tierra a una profundidad aproximada de $10 \mathrm{~cm}$ y el segundo $y$ tercero tratamiento con dos nudos bajo tierra y uno al ras del suelo, tal como se muestra en la Figura 1.

El control de malezas, se realizó manualmente durante todo el cultivo hasta la cosecha, evitando no dañar las semillas con brote. Se realizó una resiembra a los 30 días. Los riegos se realizaron con el líquido proveniente de los lavados de mandiles del establo y el agua del canal de regadío una vez por semana. Luego de la emergencia de los brotes, se aplicó fertilizantes con una fórmula inicial y única de 55-35-75 de N P K y se incorporó aproximadamente 10 toneladas/ha de estiércol de ganado bovino lechero. La cosecha se realizó a los 120 y 90 días de crecimiento al primer y tercer corte, respectivamente.

Las variables evaluadas fueron el rendimiento forrajero (t/ha), peso de planta a la cosecha (g), número de tallos a la cosecha (n) y altura de la planta a la cosecha $(\mathrm{cm})$. Se utilizó la técnica de la observación y medición para el levantamiento de la información. Para el análisis estadístico se utilizó el diseño completamente al azar y para las comparaciones de tratamientos se utilizó la prueba de Tukey. Los números de muestras tomadas fueron ochenta plantas por cada tratamiento provenientes de cuatro repeticiones.

\section{Métodos de siembra}

\section{Estacas continuas}

Inclinación $0^{\circ}$

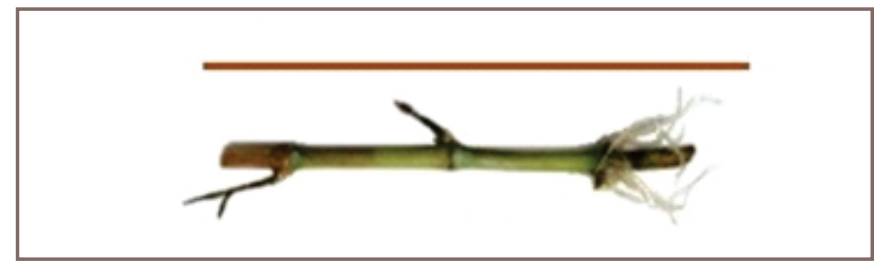

\section{Estacas continuas}

Inclinación $25^{\circ}$

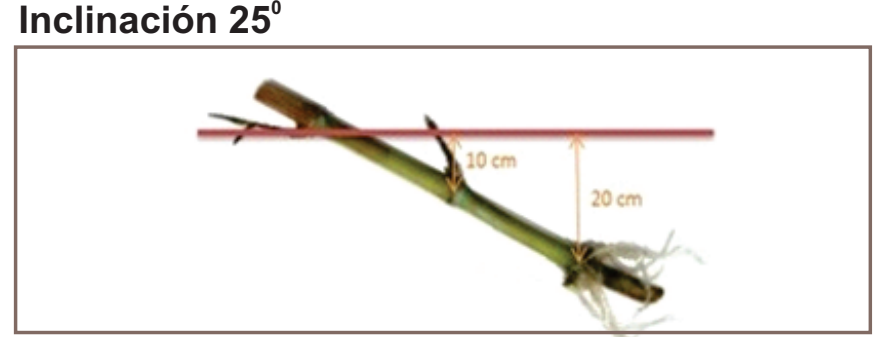




\section{Estacas continuas}

Inclinación $25^{\circ}$

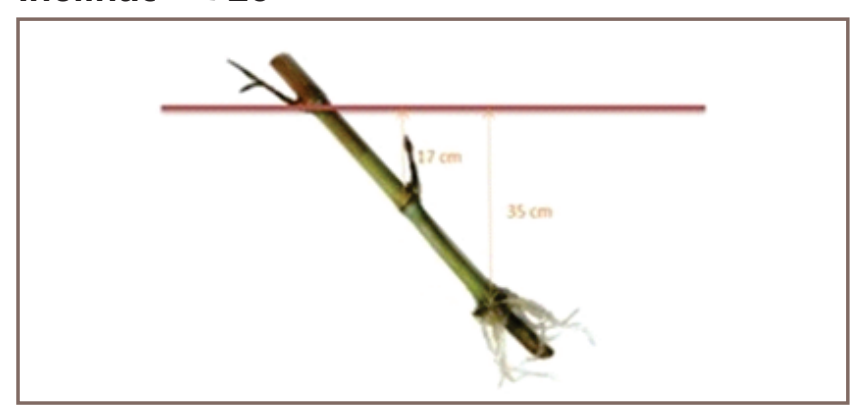

Figura 1. Métodos de siembra (grados de inclinación de las semillas en la siembra) de semillas vegetativas.

\section{RESULTADOS}

El rendimiento de forraje y el peso de la planta en el primer corte a los 120 días, el método de siembra a $25^{\circ}$ de inclinación tiene mejor resultado con $87,92 \mathrm{t} / \mathrm{ha}$ y en $6,08 \mathrm{~kg}$, respectivamente, comparado con $45^{\circ}$ y $0^{\circ}$ de inclinación $(p<0,01)$. Igualmente, en el tercer corte a los 90 días, la inclinación de $25^{\circ}$ tiene mejor resultado en el peso por planta comparado al $45^{\circ}$ y $0^{\circ}$ de inclinación $(p<0,01)$, tal como se muestran en las Tablas 1 y 2 .

Tabla 1. Rendimiento forrajero de maralfalfa según sistema de siembra y $N^{\circ}$ de corte

\begin{tabular}{lcc}
$\begin{array}{l}\text { Sistema } \\
\text { de } \\
\text { siembra }\end{array}$ & \multicolumn{2}{c}{ Rendimiento, t/ha } \\
\cline { 2 - 3 } & 1er corte & 3er corte \\
\hline $0^{\circ}$ & $61,99^{\mathrm{c}}$ & $32,02^{\mathrm{b}}$ \\
$25^{\circ}$ & $87,92^{\mathrm{a}}$ & $47,87^{\mathrm{a}}$ \\
$45^{\circ}$ & $77,78^{\mathrm{b}}$ & $33,74^{\mathrm{b}}$ \\
\hline
\end{tabular}

${ }^{a, b, c}$ Letras distintas indican diferencia estadística $(p<0,01)$.

El número de tallos por planta, tanto en el primer y tercer corte se aprecia que en el método de siembra a $25^{\circ}$ de inclinación tiene una mayor cantidad de tallos a la cosecha en comparación a $45^{\circ}$ y $0^{\circ}(p<0,05)$.

Tabla 3. Número de tallos por plantas de maralfalfa según sistema de siembra y $N^{\circ}$ corte

\begin{tabular}{lcc}
$\begin{array}{l}\text { Sistema } \\
\text { de } \\
\text { siembra }\end{array}$ & \multicolumn{2}{c}{ Tallos/planta, $\mathbf{N}^{\circ}$} \\
\cline { 2 - 3 } & 1er corte & 3er corte \\
\hline $0^{0}$ & $12,45^{\mathrm{b}}$ & $12,60^{\mathrm{c}}$ \\
$25^{\circ}$ & $15,95^{\mathrm{a}}$ & $25,80^{\mathrm{a}}$ \\
$45^{\circ}$ & $14,09^{\mathrm{ab}}$ & $21,91^{\mathrm{b}}$ \\
\hline
\end{tabular}

${ }_{a, b, c}$ Letras distintas indican diferencia estadística
$(p<0,01)$.

\section{DISCUSIÓN}

El mayor rendimiento forrajero y peso de planta observado en la siembra a $25^{\circ}$ de inclinación se debe al mayor número de tallos por planta, encontrándose mayor número de brotes en los tres nudos de las semillas vegetativas sembradas. Los rendimientos son casi similares a lo hallado por Brenes (2009), donde obtuvo 85,6 t/ha a los 70 días con buen manejo de fertilizantes, pero inferiores a los obtenido por Maguiña y Pérez (2019).

La siembra a $0^{\circ}$ de inclinación resultó con menor respuesta a los variables evaluada por presentar menor número de tallos, puede deberse a que la mayoría de las semillas brotaron en una a dos nudos y muchas de ellas no lograron emerger. Similar respuesta se observó con la siembra a $45^{\circ}$ de inclinación, con la poca o ninguna presencia de brotes del tercer nudo ubicado aproximadamente a $35 \mathrm{~cm}$ de profundidad.

El número de tallos se incrementó en el tercer corte, debido a que las plantas tienden a una mayor cantidad de tallos a medida que aumenta el número de cortes. Sin embargo, resultados superiores fueron obtenidos por Cunuhay y Choloquinga (2011), encontrado 40 tallos/planta/corte a los 120 días.

En cuanto a la altura de planta, en el primer corte lograron mejor altura que en el tercer corte, debido a la edad de la planta al momento de la cosecha. Sin embargo, la altura de planta calculada en este trabajo es inferior a lo hallado por Molina (2005)) que reporta una altura de $2,76 \mathrm{~m}$.

\section{AGRADECIMIENTOS}

Un agradecimiento especial al Sr. Raul Tomatis Chiape, por el apoyo económico y técnico en el cultivo de forraje en las instalaciones del Establo Piamonte.

\section{REFERENCIAS BIBLIOGRÁFICAS}

Ayala, J. (1990). Plantación y establecimiento. En: King grass. Plantación, establecimiento y manejo en Cuba. Ed. EDICA, Instituto de Ciencia Animal. La Habana. 34-37.

Borbor, J. (2013). Evaluación agronómica y nutricional del pasto maralfalfa (Pennisetum spp.) bajo dos métodos de propagación y tres programas de fertilización en la Parroquia Cerecita, Provincia del Guayas. Escuela Superior Politécnica del Litoral. Guayaquil, Ecuador.

Brenes, J. (2009). “Estudio técnico-económico del 
uso de diferentes tipos de fertilización en el pasto de corta Maralfalfa (pennisetum sp.)". Informe para obtener el grado de Bachiller. Instituto Tecnológico de Costa Rica. Cartago, Costa Rica.

Calzada-Marín, J., Enríquez-Quiroz, J., Hernández-Garay, A., Ortega-Jiménez, E. \& Mendoza-Pedroza, S. (2014). Análisis de crecimiento del pasto maralfalfa (pennisetum sp.) en clima cálido subhúmedo. Revista mexicana de ciencias pecuarias, 5(2), 247-260.

Cunuhay, J. \& Choloquinga, M. (2011). Evaluación de la adaptación del pasto Maralfalfa (pennisetum sp.), en dos pisos altitudinales con tres distancias de siembras en el Campus Juan Lunardi y Naste del Canton Paute. Tesis optar Titulo Ingeniero Agropecuario Industrial. Universidad Politécnica Salesiana. Cuenca, Ecuador.

Instituto Nacional Autónomo de Investigaciones Agropecuarias - INIAP. (1999). Guía de cultivos. Quito-Ecuador. 118-124.

Maguiña, R. \& Pérez, E. (2019). Número de sección del tallo para la siembra del Pennisetum sp. "maralfalfa" y el rendimiento de forraje. Infinitum. Vol. 9(1), 35.

Molina, S. (2005). Evaluación agronómica y bromatológica del pasto Maralfalfa (Pennisetum sp.) cultivado en el Valle del Sinú. Rev. Fac. Nac. Agron. Colombia, 58(1), 39.

Oquendo Lobaina, G. (2002). Tecnologías para el fomento y explotación de pastos y forrajes (No. C040.073). Agro Acción AlemanaMinisterio Federal para la Cooperación Económ ic a y DesarrolloAsociación Cubana de Producción Animal.

Correo electrónico: rufinomaximo@gmail.com

\section{Revisión de pares:}

Recibido: 15/10/2019

Aceptado: 19/12/2019 